\title{
Comparison between two models of elbow rehabilitation equipment
}

\author{
Georgiana Vetrice $^{1, *}$, and Andrea Deaconescu ${ }^{1}$ \\ ${ }^{1}$ Transilvania University of Braşov, Department of Industrial Engineering and Management, 29 \\ Eroilor Bd., 500036, Brașov, Romania
}

\begin{abstract}
International studies have revealed that using continuous passive motion (CPM) equipment as part of a rehabilitation program improves the recovery period and also diminishes the rehabilitation costs by about $50 \%$. This explains the need for conceiving elbow joint rehabilitation equipment to help persons suffering from posttraumatic disabilities. In order to create the best model to meet the patients' requirements this paper presents two constructive solutions of such equipment and a comparison between their designs. Both achieve the desired movements of the elbow joint: flexion-extension and pronationsupination and are actuated by pneumatic muscles, due to the multiple advantages of pneumatic actuation: low cost, compliance and favorable response to commands.
\end{abstract}

\section{Introduction}

The elbow joint consists of bone, cartilage, ligaments and fluid. The movement of the elbow also requires muscle and tendon input. If any of these parts is injured, the function of the elbow joint is affected. Situated between the shoulder and the hand joint, the elbow plays an important role in the functionality of the upper limb. Therefore, it is bound to be overworked and since the joint is not wrapped in layers of muscle it is prone to injuries. These traumas can be treated with or without surgery, depending on the severity of the injury. Either way, the elbow joint needs posttraumatic recovery, in order to restore its original function.

The original function of the elbow entails a movement known as flexion/extension (rotation of the forearm toward the arm and rotation of the forearm away from the arm, respectively) and another movement known as pronation/supination (rotation of the forearm by its longitudinal axis, the thumb moving closer to the body and the thumb being turned away from the body, respectively), as shown in figure 1.

In designing a posttraumatic rehabilitation program several aspects related to trauma, location and severity must be taken into account, along with other specific features of each type of trauma. Medical recovery addresses patients of all ages, suffering from various diseases and it is essential to reduce the consequences of the motor deficit on the patient's

\footnotetext{
* Corresponding author: vetrice.georgiana@unitbv.ro
} 
life. The most important method used to restore the diminished functions and increase joint mobility is physical therapy, which includes active and passive movements.

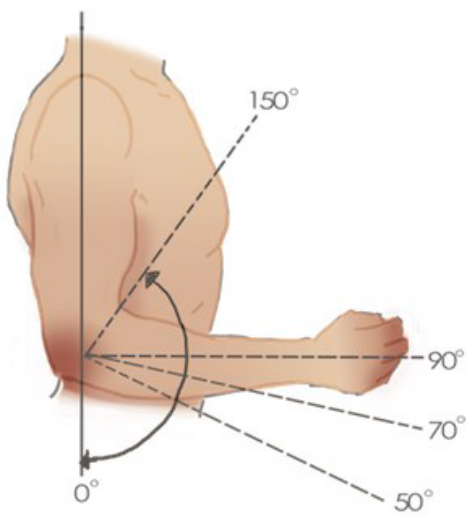

a. flexion/extension

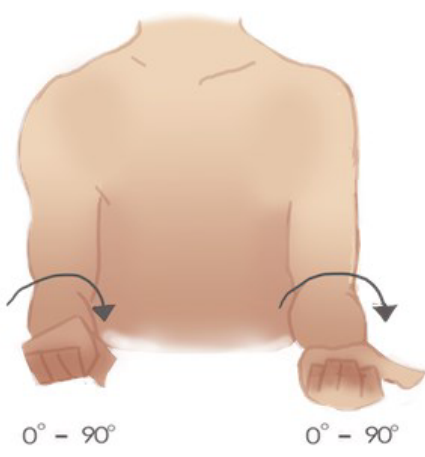

b. pronation/supination

Fig. 1. Elbow movements [1].

In case of patients that cannot achieve these movements by themselves, the recommended solution is continuous passive motion. This therapeutic procedure provides regular movement to the joint, without the tensing of the muscles.

Research has proved that rehabilitation equipment using this method is useful in the case of patients with posttraumatic disorders, improving the recovery period and reducing the quantity of pain medication.

Therefore, the rehabilitation costs are significantly reduced [2]. Although rehabilitation devices are available nowadays on the market, most of them are electrically driven and have rigid structure. This explains the need for developing new elbow rehabilitation equipment.

\section{Proposed elbow rehabilitation equipment}

\subsection{Kinematics of the rehabilitation equipment}

The equipment is modelled based on the kinematics of the human elbow joint (Fig. 2.). Therefore, the equipment will have two rotational degrees of freedom (one for flexion/extension - joint 1 and one for pronation/supination - joint 2).

Considering a secure recovery, it is very important for the rehabilitation equipment to allow for the replication of human movements, to have an ergonomic design that will minimize the discomfort of human - machine interaction, and to satisfy increased demands for safety and compliance [3]. 


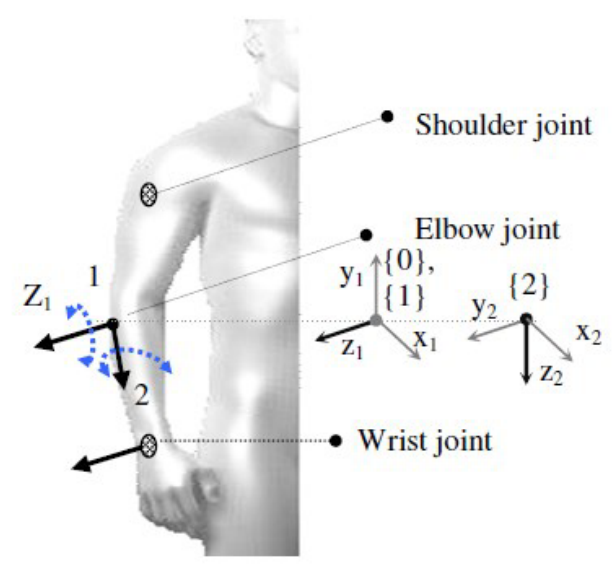

Fig. 2. Kinematics of the elbow joint [4].

\subsection{Actuating system}

Due to the specific characteristics and constructive advantages of pneumatic actuating systems, such as safe deployment, reduced overall size, easy generation, storage and transport of compressed air, the research was oriented towards the development of rehabilitation equipment actuated with pneumatic muscles.

The pneumatic artificial muscle (PAMs) is an elastic component, which increases its diameter and reduces its length under air pressure, thus carrying out a stroke (Fig. 3.a.). Pneumatic muscles are usually grouped in pairs: one agonist and one antagonist. Thus, by feeding compressed air, the pair of muscles causes the movement.

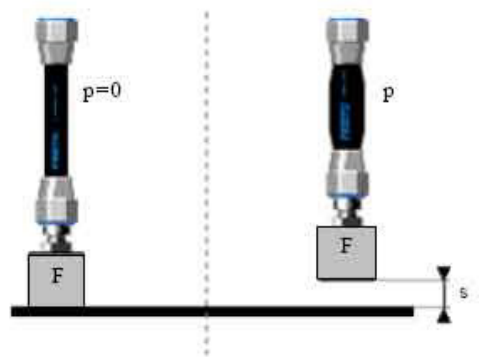

a. The stroke carried out by a PAM

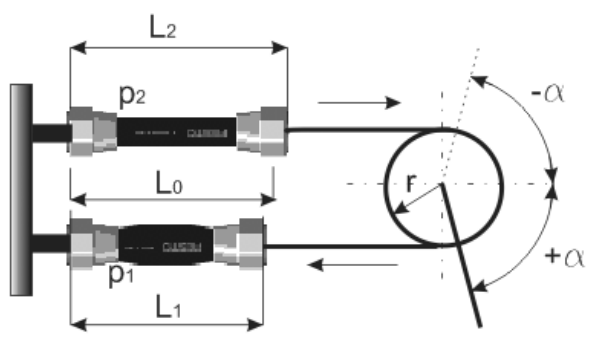

b. Kinematics of the rotation module

Fig. 3. Working principle of the pneumatic artificial muscle $[5,6]$.

The proposed equipment has two rotational degrees of freedom. Each rotation of the joint is generated by means of a pair of antagonistic pneumatic muscles. In Fig 3.b. the kinematics of such as module is shown.

\subsection{Models of elbow rehabilitation equipment}

The proposed models are shown in figure 4 and figure 5. The linkage of the equipment is similar for both rehabilitation devices. They have a hand support adjustable in length, for different sizes of the upper limb. 


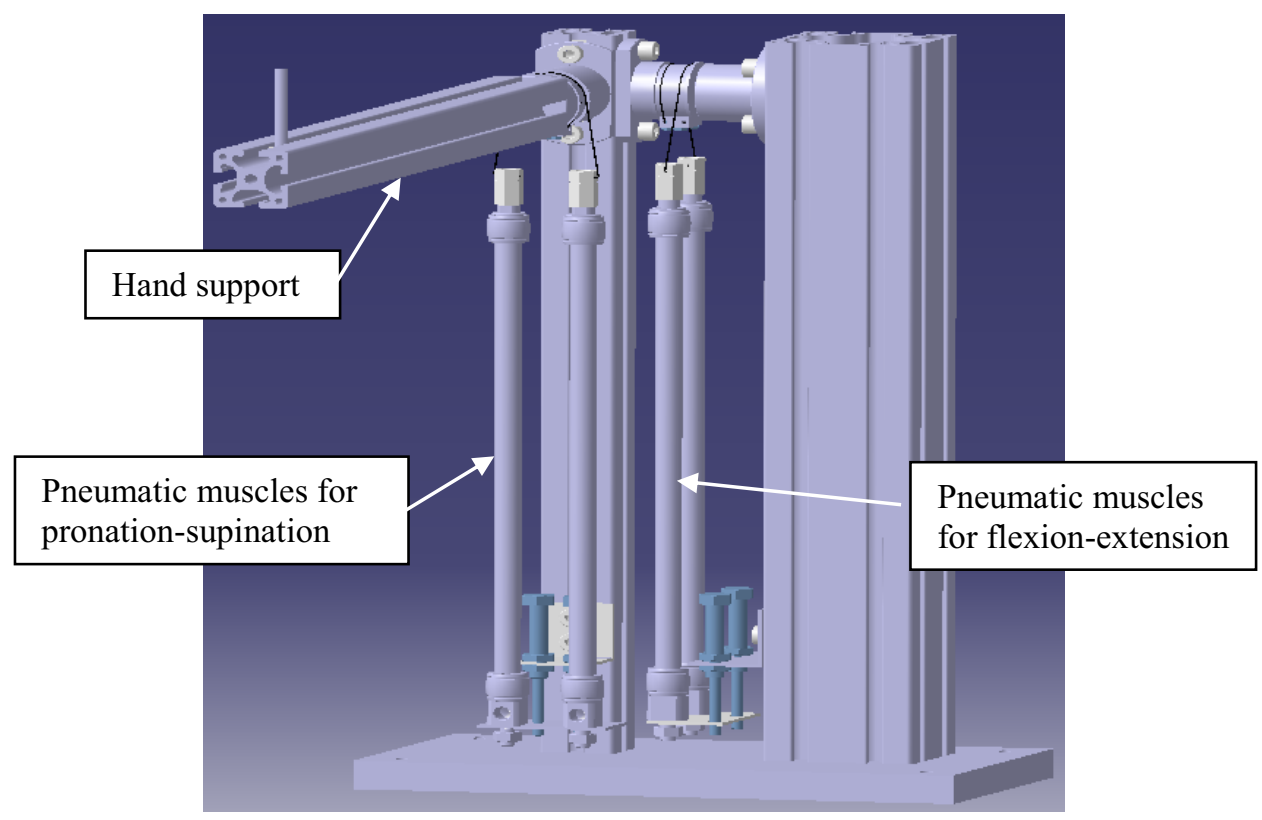

Fig. 4. First constructive variant of the elbow rehabilitation equipment.

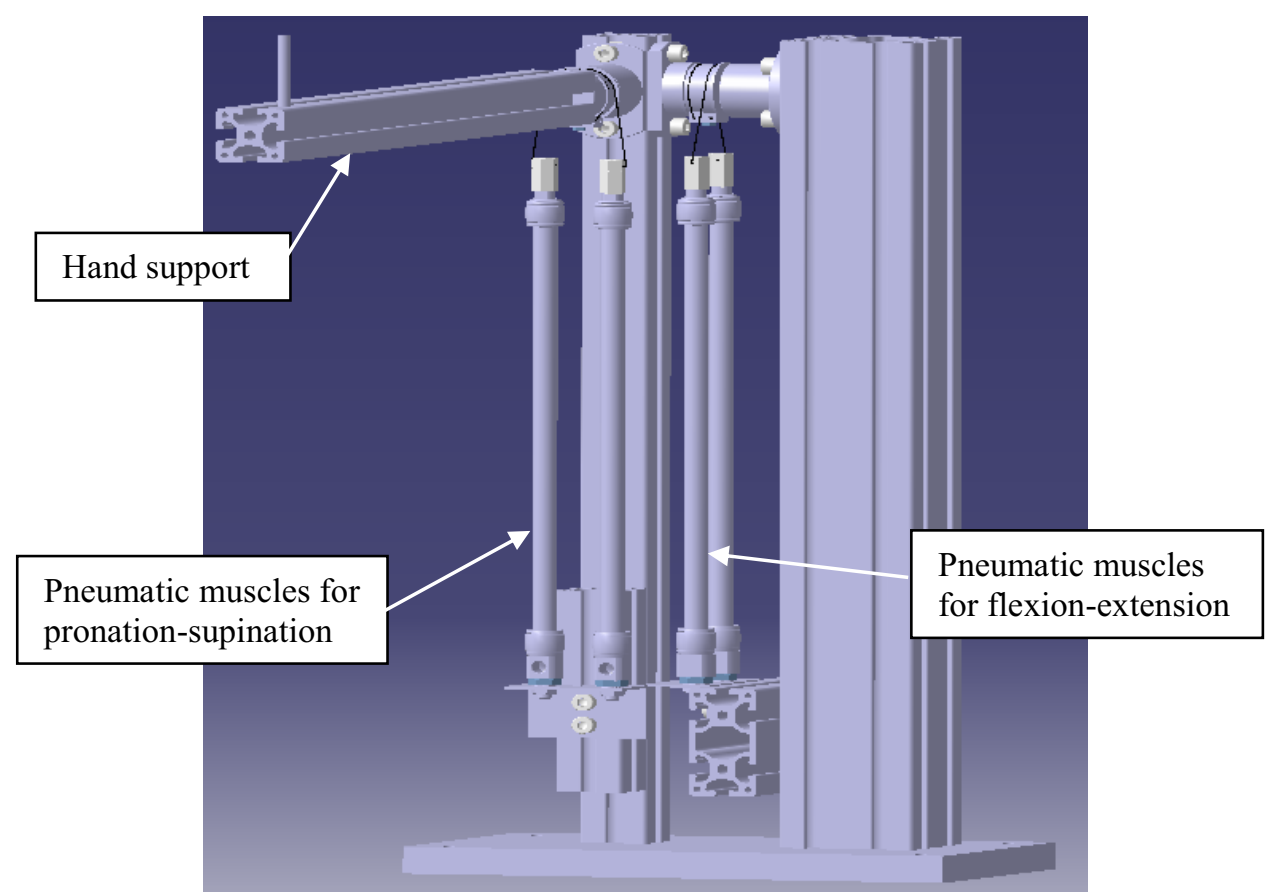

Fig. 5. Second constructive variant of elbow rehabilitation equipment.

Before rehabilitation starts, the patient places the forearm on the hand support and holds the bar. For these rehabilitation devices two pairs of pneumatic muscles were used, placed perpendicularly, one for each movement necessary to be recovered. 
For the construction of the equipment four pneumatic muscles were used, of $10 \mathrm{~mm}$ interior diameter and $300 \mathrm{~mm}$ initial length. The maximum stroke that can be achieved by these is of approximately $20 \%$ of the relaxed muscle length, which means $60 \mathrm{~mm}$.

The lower ends of the pneumatic muscles have to be fixed. For this purpose, in the first devised variant the muscles were fasten by two sheets: one tied to the metallic profile of the holding structure, the other one affixed to the free ends of the pneumatic muscles, both held together by a screw.

The second devised variant suggests fixing the lower ends of the muscles to a sheet and the sheet to the metallic profile by means of a short aluminium profile, such as to enhance stability.

In both cases, the upper ends of the pneumatic muscles are connected by a steel wire, drawn over a pulley and fixed in a specially designed structure. These wires ensure the transmission of motion between the pneumatic muscles and the joints. They are working one against the other, causing the rotation of the shaft and implicitly the rotation of the hand support. Fig. 6 presents the model of the rotational assembly.

Taking into consideration these variants, the first one was discarded and the second one maintained, namely the variant where the fixing involves a sheet and an aluminium profile, giving the equipment stability and safety in performing the movements.

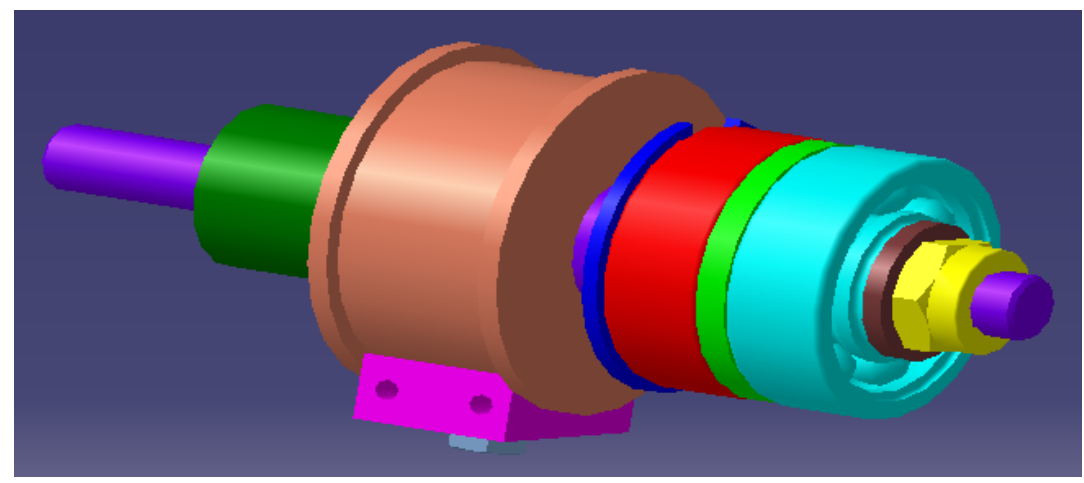

Fig. 6. The rotational assembly.

\section{Conclusions}

The upper limb is one of the most important parts of the body. Regaining the mobility of the elbow (flexion/extension and pronation/supination) is crucial for the individual's physical autonomy. Otherwise, such lack of movement results in barriers in achieving daily tasks. In the case of patients suffering from posttraumatic affections of the elbow joint rehabilitation equipment using continuous passive motion is highly eligible, as it helps reducing the recovery period and improves the efficiency of the rehabilitation process.

Although there are rehabilitation devices available on the market, these are less humanfriendly because of their electrical drives and inherent rigidity. This explains the purpose of developing new high performance and cost effective equipment for patients suffering from posttraumatic afflictions of the elbow joint.

Two variants of rehabilitation equipment of the human elbow have been developed, such as to achieve both elbow movements. The kinematics and geometric models were presented in this paper.

Both constructive variants are actuated by pneumatic muscles, due to the numerous advantages of pneumatic actuation: low weight, high power-to-weight ratio and low cost. For the movement to take place, each pneumatic muscle must have a free end and a fixed 
one. The free ends are connected to the rotational assembly by a steel wire, which ensures the transmission of the motion between pneumatic muscles and joints. The fixed ends can be fastened by two sheets or with a sheet and a short aluminium profile.

Since this equipment comes into direct contact with patients the second variant was selected, with a sheet and a profile, such as to ensure patient safety.

\section{References}

1. ***http://www.militarydisabilitymadeeasy.com/elbowandforearm.html, Accessed: 21.02.2017

2. I. Petre, T. Deaconescu, ICEEMS, IN Recent, 10, 373 (2009)

3. O. Liska, M. More, D. Janacova, H. Charvatova, MMOTE, 151 (2013)

4. M.H. Rahman, M. Saad, J.P. Kenné, P.S. Archambault, MCCA, 1567 (2010)

5. A. Deaconescu, T. Deaconescu, DAAAM, 1, 365 (2008)

6. A. Deaconescu, T. Deaconescu, IMCECS (2009) 